\title{
Bioterrorism: Preparing For The Unthinkable
}

\begin{abstract}
Terrorism is not a new concept but our need to prepare for the effects of bioterrorism has achieved a particular urgency. The use of biological agents provides a new set of challenges to professional caregivers, emergency personnel and Governments. These agents are generally not readily identified through the senses, have delayed effects and have the power to generate fear and panic. They are also intended to demonstrate that Governments and other organisations are not able to protect their citizens and members.

What evidence there is suggests bioterrorist incidents have the potential to create higher levels of psychopathology than physical injury. Therefore, the authorities must identify and rehearse suitable methods of psychoprophylaxis and intervention.
\end{abstract}

Keywords: Bioterrorism, Preparation, Training, Psychological Reactions.

\section{Introduction}

Fortunately, no society has had to endure a major bioterrorist attack. It is, therefore, hard to formulate an effective response strategy. On the other hand, there is now a genuine risk of such an event. Awareness of this risk has been emphasised by a number of factors, including intelligence confirming that Iraq had been stock piling anthrax and botulinum toxin during the Persian Gulf War, that Aum Shinrikyo, the Japanese cult, had developed its own research programme into biological weapons and had experimented with Bacillus anthracis and Clostridium botulinum toxin, and that, comparatively speaking, biological weapons are cheap and require only a modest level of scientific knowledge for their development and effective use.

By launching attacks on the general Japanese public in Matsumoto and Tokyo in 1994 and 1995 respectively, the Aum Shinrikyo breached a moral threshold. Similarly, the tragedy of the 11 th September, 2001, finally compromised any assumption that certain acts of violence would be so repellant that even the most dedicated terrorist would be inhibited from their commission.

Certain biological acts of violence may still be unthinkable but denial and reliance exclusively on intelligence and the efforts of law enforcement agencies are not adequate defences against a growing reality.

\section{The role of "terror"}

Terror has always served as a weapon against both military and civilian targets. Terror occurs when individuals are rendered helpless and hopeless and find that their customary methods of coping with adversity prove to be wholly inadequate. Also, terror occurs when one's assumptions as to what is "acceptable" are rudely and probably irrevocably compromised. History records many events in which terror has prevailed. These include the Assassins (a sect of the Shiite Muslims) of the 11-13 centuries whose victims were executed by people whom they knew and had previously trusted; Robiespierre's government owed much of its continued existence to the terrifying influence of the guillotine; the $\mathrm{Ku}$ Klux Klan waged a terror-based racist campaign in the United States of America, and the "death squads" in Argentina did much to curb dissent. Conventional terrorism, made most familiar to us by the efforts of the IRA during the "Troubles", has also posed a difficult challenge to governments and to the public. Bioterrorism, on the other hand, adds a new dimension to this challenge. Biological weapons are particularly potent sources of fear and social disruption. They have a dramatic reputation, they are not readily identifiable through the senses, and their effects may be postponed and protean. Moreover, whereas conventional weapons of terrorists, (e.g., firearms and explosive devices) usually produce most casualties in the immediate aftermath and vicinity of an attack, biological weapons may have an extended latency due to the incubation period of bacilli and viruses. The extended period of uncertainty as to whether or not someone has been infected adds considerably to the general level of anxiety.

\section{The aims of the bioterrorist}

The primary victims of bioterrorism, i.e., those who succumb to their toxic and/or incapacitating effects their agents, are only a means to an end. The main aims of bioterrorism are to create: 
- anxiety, fear and panic.

- helplessness.

- demoralisation and hopelessness.

- an extended sense of vulnerability.

- a demonstration that governments and institutions cannot protect their citizens and members.

\section{"They must be sick"}

This is a common response to any repugnant terrorist activity. Because an event may be so dreadful in its concept, a society or community will seek to explain the actions of the perpetrators as "irrational" and indicative of a disturbed mind. This may be a helpful defence; a way to explain the otherwise inexplicable. However, there is no persuasive evidence that terrorists are typically mentally ill (1), although it may be that particularly violent organisations may attract individuals with a "psychopathic" personality disorder.

However, terrorists have much to lose if they are successfully identified as mentally ill because the logic and reasonableness of their actions and cause would be called into question. To them, their actions are entirely rational and appropriate to their deeds.

\section{The strengths of the bioterrorists' strategy}

Bioterrorism can be effective for the following reasons:

- It uses a "weapon" (i.e., microbes and viruses) which cannot usually be identified through the senses and, therefore, creates uncertainty in the mind of potential victims as to their risk of being affected. This triggers a powerful and vestigial fear of mysterious and sinister forces.

- It exploits scientific and public uncertainty about the effects, particularly in the longer term.

- There is the risk that medical and other healthcare facilities and resources will be overwhelmed by the volume of victims and individuals "at risk".

- It creates unpredictability as to where the next attack might be launched, against whom, and by what means.

Because of the potency of biological weapons, they are not the weapons of "last resort"; they may be those of first choice when a weaker group needs to threaten and to intimidate a more powerful one (2).

\section{The psychological impact of bioterrorism}

Social and psychological responses to biological agents have been well documented throughout history. Records date back to the "Black Death" which killed about a third of the European population in the Middle Ages. More recently, the plague outbreak in Surat, India, demonstrated the depth of fear which a biological agent can instill (3). Nonetheless, there is still much speculation about how individuals and communities would react in the wake of a major bioterrorist event. No doubt the reactions would be partly determined by the toxicity of the agent, its incubation period and response to treatment.

\section{Panic}

Despite popular images in films and novels, and despite what the bioterrorist intends, panic is not a particularly common response even to major incidents. It is most likely in certain circumstances, however, when the individual feels trapped, and out of control, and when the opportunities for escape are clearly restricted. Such circumstances would prevail in, for example, a house fire, the sinking of a ship, and in a mine explosion (4). It is also of note that, during the Great War, the initial use of chlorine gas did provoke panic but subsequent attacks generally did not.

There may also be a paradoxical reaction (not so welcome to the bioterrorist). The terrorist activity may serve to unify and strengthen a society rather than cause it to disintegrate. It was noted that in the immediate aftermath of the terrorist bombing in Nairobi in 1998, the community (previously riven with racial, cultural and economic divisions) suddenly united and responded most constructively to this dreadful tragedy in the heart of their city (5).

\section{A classification of reactions}

It is estimated by Tyhurst (6) that, following a disaster, a community is likely to react in the following three ways. It is important that individuals, organisations and governments are aware of such reactions as crisis management will need to anticipate and to respond to them:

(i) Impact phase.

This begins when the primary stressor takes effect, and it continues until the stressor is no longer operant. During this phase, it is likely that $12-15 \%$ of the community will be able to think clearly, analyse the risks, and take constructive action. About $75 \%$ will be stunned, numb and bewildered, the remainder will be paralysed by fear, confusion or anxiety or will become "hysterical".

(ii) Recoil phase.

This phase is defined as beginning when the stressor ceases or when the individual escapes the situation. During this phase, individuals, characteristically, have a need to be with others and to talk about what has happened. It is likely that at this stage Critical Incident Stress Debriefing may be most helpful (7).

\section{(iii)Post-trauma phase.}

At this stage individuals begin to realise the full implications of what has happened, what they have lost and 
suffered, and what will be facing them in the future. It can also be a period of disappointment when promises are not fulfilled and when there appear to be delays in needs being met.

\section{Individual reactions over time}

Obviously, some individuals may react in entirely idiosyncratic ways following a bioterrorist outrage, but below are some typical psychological responses:

- horror.

- anxiety and fear (but, as stated above, panic is not common).

- anger (at the terrorists or agencies of Government if their reactions are perceived to be inadequate or delayed).

- scapegoating (after trauma there is often a powerful need to blame somebody).

- paranoia (a powerful sense of persecution and vulnerability).

- demoralisation and helplessness (individuals are not sure what to do to help themselves or their loved ones).

- social isolation and stigma (this is particularly likely if quarantine and decontamination are required. See "Secondary trauma" below).

- loss of faith (trauma shatters assumptions about various matters including the ability of the State or organisations to keep us safe and secure).

- grief (trauma always involves loss of some kind).

- guilt (some may feel guilty for having come through the attack when others did not (i.e., "survivor guilt"), whereas others may feel guilty for not having done enough to help others (i.e., "performance guilt")).

- "magical thinking" (this reflects usually rather primitive ways of thinking about the biological agents and how to combat them).

- false attributions (normal stress reactions, e.g., hyperarousal may be attributed to the biological agent).

- xenophobia (after the bombing of the Murrah Federal Office building in Oklahoma, there was a widely prevalent belief that Arab terrorists had been responsible).

\section{Sociogenic illness}

Whilst panic is not common after trauma, it is common for individuals to present with symptoms which do not appear to have any physical basis (8). This phenomenon has been called "sociogenic illness" or "medically unexplained physical symptoms". The most common examples of such symptoms are nausea, vomiting, dizziness and light-headedness. There are numerous reports of this phenomenon. DiGiovanni (9) describes a number of these. During World War I, at one referral centre 281 soldiers were admitted following a gas attack but only 90 had been genuinely contaminated by gas, the others were suffering from "gas mania". Following the sarin attack in the Tokyo subway by Aum Shinrikyo 5510 persons sought treatment. Twelve died; 17 were critically injured; 1370 had moderate injuries, and the remainder had minor or no injuries. Following a missile attack on Israel by Iraq during the Persian GulfWar nearly $40 \%$ of the civilians in the vicinity of the attack reported breathing difficulties, tremor, sweating and anxiety. Significantly, however, subsequent attacks produced fewer symptoms.

A major implication of these observations is that physicians and mental health personnel need to be trained to distinguish between those symptoms which are of biological origin from those which are of psychological origin - not an easy task!

\section{The "worried well"}

This is a related concept; the validity of which has been challenged by Pastel (4). He argues that this is a misnomer because these individuals have a right to be worried and they are not "well" because their suffering and anxiety is genuine. Nonetheless, the term is intended to describe those individuals who are concerned about their health following exposure to some kind of trauma. An obvious example was the thousands of Vietnam Veterans who sought investigations and examinations for the effects of "Agent Orange" (dioxin). Similar reactions were noted after the $\mathrm{El} \mathrm{Al}$ airline crash in Amsterdam in 1992 (10) and after the 3 Mile Island nuclear accident (11).

These related phenomena of the "sociogenic illness" and "worried well" alert us to the fact that, following a major bioterrorist incident, there would be a likelihood that the medical and healthcare services would be overwhelmed by the numbers of casualties - physical and psychological. Any strategic plan would need to consider how to deal with this circumstance, particularly if the ratio of physically injured to non injured were about 1 to 500 as has been estimated $(2,12)$.

\section{Secondary trauma}

Paradoxically, steps taken by the authorities following a bioterrorist incident could themselves become a source of genuine distress to the survivors:

- There may be a significant delay before the significance of the incident is acknowledged and before the active agents have been identified (as occurred after Chernobyl).

- Medical interventions may cause iatrogenic illness and distress. (After the Tokyo sarin attack, there was much concern about the fact that there were inadequate showering facilities; little attention was paid to modesty and to privacy in relation to individuals having to 
be undressed and washed with bleach, and personal clothing had to be destroyed.)

- Quarantine and isolation may disintegrate family and social supports.

- Living in unfamiliar and bizarre barrier environments can lead to individuals feeling stigmatised. There would be the problem of relocation if individuals could not be returned to their infected homes/areas.

- There may be publicly expressed disagreement among experts as to what has happened and what should be done to mitigate the effects of the incident.

\section{The media}

The involvement of the media following a bioterrrorist event is a cardinal issue as confirmed by Quigley (13). Because of the advances of modern information technology and communication networks, information (and, indeed, misinformation) can be transmitted almost instantaneously. Media personnel can even arrive at the scene of an incident before the emergency services.

The media are of course important to the terrorists as they provide the terrorists with an opportunity to give a wide airing to their "message" and cause. More specifically, the terrorists will hope to persuade through the media that there is a moral rationale to their activities, even the most extreme ones.

On the other hand, the authorities can use the media to ensure that accurate information is relayed to all those implicated in the event. The key point is "accurate" since rumours abound after terrorist incidents. The authorities should decide not only what information should be disseminated but by whom. Credible presenters are essential. Information which is found to be useful after traumatic events includes an accurate representation of what has happened; what is happening; what will happen; what resources and help are available, and what individuals can do to help themselves.

Two important considerations for the authorities in terms of what to communicate are that: (i) individuals are less influenced by statistics than they are by case studies, and (ii) they are more influenced by perceived outcomes than by statistical probabilities (14).

\section{The organisational response to psychological needs}

Several authorities have outlined the technical response to specific agents (e.g. Arnon et al (15), in relation to botulinum toxin, and Ingleby et al (16), in relation to anthrax), but it is now widely recognised that there needs to be a systematic and well rehearsed response to the psychological needs of those affected by a bioterrorist event.
How a government or organisation responds in the wake of a terrorist incident is crucial. Ill considered or inappropriate reactions may fuel anxiety and distress and, inadvertently, may encourage support for the terrorists' ideals and aims. Everly and Mitchell (17) have outlined the 10 commandments of responding to mass terrorist attacks.

In outline, these are as follows:

(i) A terrorist act aims to achieve psychological instability.

(ii) Establish crisis intervention hot-lines and walk-in crisis centres.

(iii) Provide adequate psychological preparation before, and support during and after an incident for emergency services.

(iv) Collaborate with the media.

(v) Re-establish a sense of physical safety for the public.

(vi) Enlist the support of local political, educational, medical, economic, and religious leaders to facilitate communication with general public.

(vii) Re-establish, as soon as possible, normal communication, transport, education and work schedules.

(viii)Use symbols to re-establish community cohesion (e.g., flags, car stickers, and posters).

(ix) Initiate rituals to honour survivors, rescuers and the dead.

(x) Do no harm.

Another model of intervention is "psychological first aid", first enunciated by Raphael (18) and developed by others (e.g. Alexander (19)). This concept aims to minimise suffering, reduce anxiety and fear, impose order and structure, and harness the healing potential of ordinary citizens and their families. Some of the key elements in this strategy are as follows:

- Provision of comfort and protection (often in their distress and despair, trauma victims may do things which make matters worse for themselves).

- Provide (accurate) information (in almost any intervention strategy accurate information plays a key role).

- Re-establish order (this can be conveyed in various ways, for example, the reinstitution of power, transportation, and communication services. Even the humble cup of tea (whatever its intrinsic restorative properties!) conveys that the world is again under some degree of control and order).

- Engage purposefully (ensuring that victims have something purposeful to do in the immediate aftermath of an incident may serve as an antidote to the otherwise disturbing sense of helplessness, and it may reduce the level of guilt commonly reported after trauma - see Individual reactions over time).

- Facilitate links (these links may be with 
other members of their family, group or community who would be in a position to offer support and succour).

- Conduct "triage" (no two individuals react in exactly the same way following the same trauma. Individuals will vary in terms of their vulnerability depending on their personality, the way they perceive the trauma and their personal circumstances, see (20). It is important, therefore, to identify those at greatest risk and for whom the limited mental health resources should be made available).

Obviously, for reasons stated above, defining a strategy of response following a bioterrorist event is no easy task. However, what is important is that strategies are defined and are tested rigorously in realistic exercises with all the relevant "players" actively involved.

\section{Who cares for the carers?}

Alexander and Atcheson (21) have already confirmed that those who provide care for victims of trauma may themselves be at risk of adverse emotional reactions as a direct consequence of their work. Involvement in a major bioterrorist incident is likely to put very special pressures on emergency and healthcare personnel. It would be reasonable to assume that those who are the "first responders" may well be subject to the same psychological reactions as ordinary citizens, despite their training and natural fortitude. However, in addition, they may be faced with another challenge, namely, having to wear protective equipment. "Gas mask phobia" was recognised during World War I. Individuals thus affected experienced panic, an inability to breathe, sweating and hyperventilation (22). Also, during the Gulf War some service personnel had to be evacuated from the field of conflict because of their inability to tolerate protective respirators. It is now also recognised that wearing protective clothing can create adverse emotional reactions and compromise function (physical and psychological) in various ways as described by Krueger (23).

On the other hand, it is obviously essential that first responders are properly attired and equipped. During the sarin incident in Tokyo, the Tokyo Fire Department sent 1364 personnel to deal with the incident. Of these 135 (i.e., approximately 10\%) were themselves subject to contamination by the sarin (24).

DiGiovanni (11) has outlined a number of important issues which need to be addressed by those who respond following a bioterrorist incident. These are:

- training (with realistic scenarios involving "psychological" as well as physical casualties).

- dealing with the implications of a policy of quarantine or at least of voluntary restriction on travel.
- establishing an effective liaison with the media.

- a public education campaign.

- a policy on how to incorporate all relevant personnel, including mental health specialists.

According to DiGiovanni (9) mental health personnel would have a number of important functions including:

- advising authorities about the management of an anxious and distressed population.

- offering information and guidance to medical and surgical staff.

- providing support for healthcare and emergency personnel.

- assessing the mental health status of primary victims, and

- conducting triage (particularly to identify those whose symptoms are due to biological factors and those due to stress and anxiety).

DiGiovanni (11) also highlights the importance of establishing a clear policy on how to deal with dead bodies, as this factor is likely to be a highly sensitive one which may influence how well the families of the deceased adjust to the event. Infection and contagion raise important questions about access to the bodies of the deceased and about the disposal thereof. It needs to be remembered that their cultural sensitivities and religious prohibitions (with regard to cremation, for example) need to be considered in relation to public health requirements.

\section{Conclusions}

(i) Bioterrorism represents a new and significant threat to health and social stability. We are only beginning to formulate how best to respond to and to combat this threat. We do not have the luxury of an extended period of trial and experimentation.

(ii) The risk of a major bioterrorist incident can no longer be dismissed as a paranoid fantasy or an extravagant piece of fiction.

(iii)Information and guidance have to be gleaned from other forms of trauma, including chemical accidents and terrorist activity involving chemical agents.

(iv) Any organisation or government which does not define a preventative and intervention strategy has to be considered incompetent.

(v) Effective preventative and intervention strategies need to be multi-professional, well rehearsed and well coordinated.

(vi) One of the most powerful antidotes to the fear, anxiety and uncertainty which bioterrorist activities seek to create is accurate information. Thus, the media can play a major role, and the authorities need to determine how to work constructively with the media. 


\section{References}

1. Reich W. Understanding terrorist behavior. In: Reich W ed. Origins of terrorism: Psychologies, ideologies, theologies, states of mind. Cambridge: Cambridge University Press 1990.

2. Knudson GB. Nuclear, biological, and chemical training in the U.S. army reserves: Mitigating psychological consequences of weapons of mass destruction. Military Medicine 1990; 166: 63-65.

3. Ramalingaswami V. Psychosocial effects of the 1994 plague outbreak in Surat, India. Military Medicine 2001; 166: 29-30.

4. Pastel RH. Collective behaviors, mass panic and outbreaks of multiple unexplained symptoms. Military Medicine 2001; 166: 44-46.

5. Alexander DA. Nairobi terrorist bombing: The personal experience of a mental health adviser. Int f Emergency Mental Health 2001; 3: 5-13.

6. Tyhurst JS. Individual reactions to community disaster: The natural history of psychiatric phenomena. Am f Psychiatry 1951; 107: 764-769.

7. Simon JD. Nuclear, biological, and chemical terrorism: Understanding the threat and designing responses. Int F Emergency Mental health 1999; 1: 81-89.

8. Wessley S, Hymans KC, Bartholomew R. Psychological implications of chemical and biological weapons. Br Med J 2001; 323: 878-879.

9. DiGiovanni C. Domestic terrorism with chemical or biological agents. Psychiatric aspects. Am $\mathcal{f}$ Psychiatry 1999; 156: 1500-1505.

10. Engel CC. Outbreaks of medically unexplained physical symptoms after military action, terroristthreat, or technological disaster. Military Medicine 2001; 166: 47-48.

11. DiGiovanni C. Pertinent psychological issues in the immediate management of a weapons of mass destruction event. Military Medicine 2001; 166: 5960.

12. Salter CA. 2001. Psychological effects of nuclear and radiological warfare. Military Medicine 2001; 166: $17-18$.
13. Quigley C. Dual-edged sword: Dealing with the media before, during, and after a weapons of mass destruction event. Military Medicine 2001; 166: 5658 .

14. American Psychological Association. Handling anxiety in the face of the anthrax scare. APA HelpCenter: Get the facts: Psychology in Daily Life 2001. http://helping.apa.org/daily/anthrax. html

15. Arnon SS, Schechter R, Inglesby $\mathrm{TC}$ et al. Botulinum toxin as a biological weapon. $\mathscr{f} A M A$ 2001; 285: 1059-1070.

16. Insglesby TV, Henderson DA, Bartlett JG et al. Anthrax as a biological weapon: med \& public health management. FAMA 1999; 281: 1735-1745.

17. Everly G, Mitchell JT. America under attack: The "10 Commandments" of responding to mass terrorist attacks. Int $\mathcal{f}$ Emergency Mental Health 2001; 3: 133-135

18. Raphael B. When disaster strikes: How individuals and communities cope with catastrophe. New York: Basic Books 1986.

19. Alexander DA. Human reactions to trauma. In: Greaves I, Porter K eds. Pre-Hospital medicine. The principles and practice of immediate care. London: Arnold 1999.

20. Alexander DA. Psychological aspects of trauma. In: Greaves I, Porter K, Burke D eds. Key topics in trauma. Oxford: Bios Scientific Publications 1997.

21. Alexander DA, Atcheson SF. 1998. Psychiatric aspects of trauma care: a survey of nurses and doctors. Psychiatr Bull 1998 22: 132-136.

22. Ritchie EC. Psychological problems associated with mission-oriented protective gear. Military Medicine 2001;166: 83-84.

23. Krueger GP. Psychological and performance effects of chemical-biological protective clothing and equipment. Military Medicine 2001;166: 4143.

24. Tucker JB. National health and medical services response to incidents of chemical and biological terrorism. $\mathcal{F} A M A$ 1997; 278: 362-368. 